October 31, $2018 \quad$ 7:57 WSPC/INSTRUCTION FILE Homan`1

International Journal of Modern Physics: Conference Series

(C) World Scientific Publishing Company

\title{
Physical Properties of Jets in AGN
}

\author{
Daniel C. Homan \\ Department of Physics and Astronomy, Denison University \\ Granville, OH 43023, USA homand@denison.edu
}

Received Day Month Year

Revised Day Month Year

\begin{abstract}
I review constraints on the physical properties of AGN jets revealed through Very Long Baseline Interferometry (VLBI) studies of the structure and time-evolution of parsecscale jets, including recent results from the MOJAVE program. In particular I focus on constraints available from very long time baseline studies which probe a wide range of jet behavior over many outbursts. Kinematic studies of propagating jet features find an apparent speed distribution that peaks around $10 c$ for blazars, with speeds up to $50 c$ observed. These observed speeds require Lorentz factors at least as large, implying that parsec-scale Lorentz factors up to $10-20$ are common for blazars with a tail up to $\sim 50$. Jet flows are still becoming organized on these scales as evidenced by the high incidence of non-radial motions and/or accelerations of jet features (including increases and decreases in apparent speed and direction). Changes in Lorentz factors of propagating jet features appear to play a significant role in the observed accelerations, and while the connection between acceleration of jet features and the underlying flow is not clear, the pattern of observed accelerations suggest the flow may increase in speed near the base of the jet and decrease further out. In some jets, ejections of new features span a range of ejection angles over many epochs, tracing out wider opening angles on parsec-scales than are apparent in single epoch observations.
\end{abstract}

Keywords: Keyword1; keyword2; keyword3.

PACS numbers: $11.25 . \mathrm{Hf}, 123.1 \mathrm{~K}$

\section{Introduction}

A key goal of Very Long Baseline Interferometry (VLBI) studies of AGN jets is to address long standing questions about jet formation, collimation, and acceleration 1 - 4 . These studies also seek to understand the distribution of intrinsic jet power and speeds 51617 and the connection between those properties and other high energy phenomena 8 -11. VLBI observations of AGN jets directly probe the structure, polarization, and kinematics of extra-galactic radio jets on parsec scales. Because jets are moving very close to the speed of light and directed close to our line of sight, their emission is highly Doppler boosted, and features moving within the jets exhibit apparent superluminal motion: 


$$
\beta_{o b s}=\frac{\beta \sin \theta}{1-\beta \cos \theta}
$$

where $\beta$ is the intrinsic speed of the moving feature and $\theta$ in the angle its motion makes with our line of sight. For a given $\beta$, this motion is a maximum when $\beta=$ $\cos \theta$, in which case $\beta_{o b s}=\beta \Gamma$ where $\Gamma=1 / \sqrt{1-\beta^{2}}$ is the Lorentz factor of the moving feature.

An important question is the extent to which any single jet feature or 'component' is characteristic of the jet flow. It is now commonly believed that most jet features represent propagating oblique or transverse shocks in the flow 12 . Consistent with this picture, a wide range of behavior is seen among components within a single jet, including stationary or quasi-stationary features $16-21$, although it is important to note that Ref. 21 found the distribution of apparent speeds within individual jets to be significantly narrower than between different jets, suggesting that individual jets do have a characteristic flow speed. When multiple speeds are seen in a single jet, slower speeds may represent trailing shocks 14 , and therefore the best estimate of the flow speed may be the fastest component observed 21 .

The National Radio Astronomy Observatory' a Very Long Baseline Array (VLBA) has been in continuous operation since 1994, allowing regular monitoring of large samples of AGN radio jets, with some individual jets having regular monitoring observations for 16 years and counting. The large sample sizes and very long time baselines allowed by the continuous operation of the VLBA has made it possible to study AGN jets in entirely new ways to address these questions. In the following sections I will discuss the impact on our understanding of (1) the distribution of apparent jet speeds and intrinsic Lorentz factors, (2) the acceleration and collimation of jets, and (3) the morphology and opening angles of parsec-scale jets.

\section{Long Time Baseline VLBI}

As described above, the VLBA has made it possible to study both large samples of AGN jets and to continuously monitor jets over long time intervals. The longest running example of this kind of program is the $2 \mathrm{~cm}$ Survey/MOJAVE program. MOJAVE stands for Monitoring Of Jets from Active galactic nuclei with VLBA Experiments 22 , and it is a continuation of $2 \mathrm{~cm}$ Survey which started in 199425/26/17/27. The MOJAVE program currently images the parsec scale structure and polarization of more than $300 \mathrm{AGN}$ jets at $15 \mathrm{GHz}(\lambda 2 \mathrm{~cm})$ on a regular basis. Many of the jets in the 135 source MOJAVE-I complete, flux-density limited sample 24 have observations spanning more than 15 years. The Gamma-ray AGN monitoring project at Boston University has focused on monitoring a smaller number of Gamma-ray Blazars identified by EGRET and FERMI at higher frequencies

a The National Radio Astronomy Observatory is a facility of the National Science Foundation operated under cooperative agreement by Associated Universities, Inc. 
(22 and $43 \mathrm{GHz}$ ) with more closely spaced epochs $28|18| 11$. While it is difficult to track individual jet features over long periods of time at these higher frequencies, several of the AGN in their program also have data spanning more than 15 years. At lower frequencies $(2$ and $8 \mathrm{GHz}$ ) the Radio Reference Frame Image Database (RRFID) has images spanning a similar period of time 29 ; however, only a small window from 1994-1998 has been analyzed for kinematics 19 .

Figure 1 is an example of the kind of the kinematic data that can be obtained by observing a parsec scale jet over long periods of time. The TeV Blazar $1222+216$ has $15 \mathrm{GHz}$ monitoring observations going back to 1996 as part of the Brandeis University monitoring project $\frac{1630}{30}$ and has been observed as part of the $2 \mathrm{~cm}$ Survey/MOJAVE program since 1999. The MOJAVE program identifies several moving components that can be tracked throughout much of this period 21 , and the motion of component " 5 " is illustrated in the figure. It has an apparent motion nearly $17 c$ and makes a distinct bend in its trajectory to the East, magnified by projection to appear to be a $40^{\circ}$ change in the plane of the sky 31 .

\subsection{Distribution of Apparent Speeds}

By studying the apparent speed distribution of a large, flux-density limited sample of AGN jets we probe the underlying Lorentz factor distribution of the parent population 56 . This assumes that the apparent speeds of moving jet features are good tracers of the underlying flow velocity, and as discussed in the introduction, the range of apparent speeds seen in individual objects suggests that we should use only the largest apparent speed observed in a given jet. Thus it is necessary to observe jets long enough to sample multiple components to increase our confidence that we have seen a component characteristic of the flow speed. In their analysis of the 135 source MOJAVE-I complete sample, Ref. 21 obtained motions for 127 AGN jets and found that the distribution of the fastest component in each jet peaked around $10 c$ with a tail extending up to $50 c$. Analysis of the effects of beaming on flux-density limited samples show that for a large sample, like the MOJAVE-I sample, the fastest observed speed in the sample is characteristic of the maximum Lorentz factor in the parent population $\underline{6}$; thus, Ref. 21 concludes that the intrinsic Lorentz factor distribution is likely a power-law distribution with a tail extending up to $\Gamma \simeq 50$. It is interesting to note that a jet beamed directly at us has a Doppler factor $\delta=(1+\beta) \Gamma$, so Doppler factors of up to $\sim 100$, although rare, may be observed in the blazar population.

Ref. 18 find a similar range of speeds in their sample of 15 blazars at $\sim 3 \times$ higher resolution, suggesting that the much larger MOJAVE sample is indeed sensitive to the fastest jet speeds emerging from the core region, despite the resolution difference. Ref. 18 also performed an additional analysis of the apparent fading times of the jet components they followed in an attempt to obtain an independent estimate of the Doppler factor for individual components. The combination of Doppler factor and apparent speed for individual components allowed a direct estimate of the Lorentz 


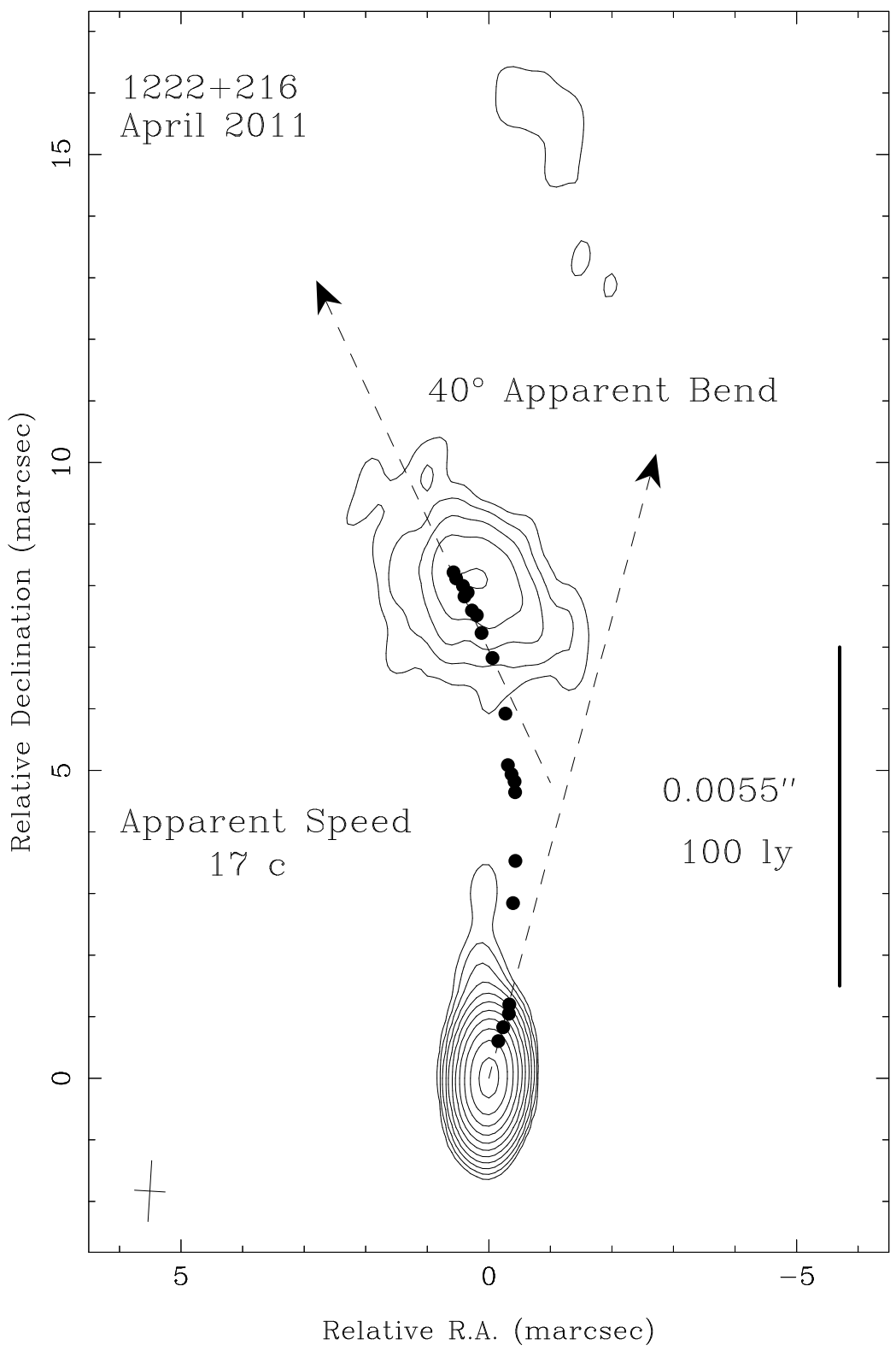

Fig. 1. Plot of 12 years of MOJAVE position data for jet component " 5 " in the TeV Blazar $1222+216$ at $z=0.434$. The component has an apparent speed of nearly $17 c$, and shows a marked bending of its trajectory to the East. The positions are superposed on a $15 \mathrm{GHz}$ MOJAVE VLBA image from April 2011 (http://www.physics.purdue.edu/astro/MOJAVE/). Contours begin at 1.5 $\mathrm{mJy} /$ beam and increase in factor of two steps. 
factors for those components which fell in the range $\Gamma=5-40$.

The lower frequency Caltech-Jodrell Bank Flat-Spectrum (CJF) sample of speeds in $237 \mathrm{AGN}$ at $5 \mathrm{GHz} 20$ and the RRFID kinematic analysis at $8 \mathrm{GHz}$ of 77 sources 19 have distributions that peak at lower speeds with tails up to $\simeq 30 \mathrm{c}$. At a factor of 2-3 times lower spatial resolution, these studies sample jet emission further from the core region. So the speed difference may be due to jets already starting to slow down at these length scales, or perhaps the jet components that survive to these distances are systematically different than those closer to the core.

\subsection{Acceleration and Collimation}

Some models of jet formation and collimation have jets being fully accelerated and collimated very near the super-massive black hole/accretion disk system on length scales much smaller than those probed by $\mathrm{VLBl}^{3}$ while other models extend this process of much larger length scales 2 where VLBI observations may be able to see acceleration in action. It is also unknown where jets begin to slow down before they reach kiloparsec scales. Very long time baseline VLBI studies have the opportunity to address these issues by tracking individual jet components over a very large number of epochs. We can study acceleration by looking for changes in the apparent velocity vector $\vec{\beta}_{o b s}$ as described in Ref. 32;

$$
\begin{gathered}
\frac{d \beta_{\| o b s}}{d t_{o b s}}=\frac{\dot{\beta} \sin \theta+\beta \dot{\theta}(\cos \theta-\beta)}{(1-\beta \cos \theta)^{3}} \\
\frac{d \beta_{\perp o b s}}{d t_{o b s}}=\frac{\beta \dot{\phi} \sin \theta}{(1-\beta \cos \theta)^{2}},
\end{gathered}
$$

which are the parallel and perpendicular components of the observed acceleration on the sky. $\dot{\beta}, \dot{\theta}$, and $\dot{\phi}$ are the intrinsic rates of change of the component speed, angle to the line of sight, and azimuthal angle respectively.

If observed parallel accelerations are due entirely to changes in the component's Lorentz factor, $\Gamma$, there is a simple relation with the observed quantities 32 .

$$
\frac{\dot{\Gamma}}{\Gamma}=\frac{\dot{\beta}_{\| o b s}}{\beta_{o b s}} \frac{\beta^{2}}{\delta^{2}}
$$

where the Doppler factor $\delta=1 /(\Gamma(1-\beta \cos \theta))$. If $\delta$ can be estimated, this approach allows one to measure the ratio $\dot{\Gamma} / \Gamma$ which can be compared to physical models for acceleration or deceleration.

A key question is the extent to which apparent accelerations in jet motions can be assigned to changes in the Lorentz factor of the jet feature or changes in the angle to the line of sight. In their kinematic analysis of the $2 \mathrm{~cm}$ Survey, Ref. 17 
found that $30 \%$ of the jet components they studied for non-radial motion were indeed moving on a vector mis-aligned with the radio core, indicating that these components had changed their trajectory since being ejected from the base of their jet. In general these "non-ballistic" jet features were misaligned toward the direction of the next structure in the jet, indicating that they were following pre-established channels 17 . These results were confirmed by both the RRFID kinematic analysis 19 and the MOJAVE kinematic analysis, which extended the time baseline of the $2 \mathrm{~cm}$ Survey by several years 21 . When only the most well determined MOJAVE jet components were analyzed (those suitable for acceleration analysis) the fraction with non-ballistic motion increased to nearly $50 \% 32$.

The long time baseline and very large number of epochs in the MOJAVE program allowed Ref. 32 to extract the 203 best best studied jet features in their sample for direct acceleration analysis. They measured the apparent parallel and perpendicular accelerations. Parallel accelerations are along the component motion, indicating changes in apparent speed, and perpendicular accelerations indicate changes in direction. By studying the ratio of these two quantities, they concluded that intrinsic changes in the Lorentz factor of jet components were common 32 . They observed a tendency for jet components with increasing apparent speed to be closer to the base of their jets than components with decreasing apparent speed, suggesting that the jet flow may increase in speed near the base of the jet and decrease further out ${ }^{32}$, although this assumes the observed pattern changes are reflective of the underlying flow. Ref. 18 also observed a tendency for positive acceleration of apparent speeds near the base of jets in their sample of 15 blazars at $43 \mathrm{GHz}$. While the authors interpreted these results as evidence that the jets were bending away from the line of sight (and closer to the optimum angle for superluminal motion) 18 , the MOJAVE program results described above suggest that these changes may be better explained (on average) by increases in the Lorentz factors of those components.

\subsection{Jet Opening Angles and Morphology}

Ref 39 used the most recent MOJAVE epochs available at the time to study the correlation between apparent jet opening angle and Gamma-ray brightness of AGN jets. They found that Gamma-ray blazars had significantly larger apparent opening angles than non-detected AGN, indicating that these Gamma-ray bright AGN are more closely aligned with the line of sight and therefore more highly beamed 39 . They also combined their apparent opening angle results with Doppler factor measurements from Ref. 40 and apparent speeds from the MOJAVE program 21 to extract intrinsic opening angles, finding average jet opening angles on parsec scales of $1.2 \pm 0.1$ degrees for quasars and $2.4 \pm 0.6$ degrees for BL Lacs.

It has been well known for some time that some of the best studied blazar jets show ejections of new jet features at multiple position angles $33-38$. The very long time baselines in the MOJAVE program have allowed us to sample a wide range of component ejection and propagation behavior in jets. For jets with a range 
of ejections angles, the structure at any single epoch may only show those areas of the jet that have been recently illuminated by a passing component. This can give the false impression of bent or twisted jet trajectories when the component motion is actually largely ballistic. An example is shown in figure 2, where a single epoch image of the quasar $1308+326$ is compared to a 'stack' of 58 epochs from the MOJAVE program 24 . The single epoch image gives the impression of a sharply bent trajectory, whereas the reality is that the features are moving outwardb in a broad cone on the sky which is revealed in the stacked image. Images of this kind suggest a possible new way to study jet opening angles and collimation through time-averaged morphology over the course of many outbursts.

\section{Conclusions}

Very Long Baseline Interferometry studies of parsec-scale jets with structural and polarization information gathered over very long time baselines are producing interesting new results. By observing multiple jet features over long periods of time, we are able to obtain better estimates of maximum apparent jet speeds in individual jets and use these when studying the Lorentz factor distribution of AGN jets as a whole. Lorentz factors up to 10-20 appear to be relatively common in blazar jets with a tail extending up to a maximum Lorentz factor of $\sim 50$ for the blazar population $18 \mid 21$. When individual jet features are tracked for a large number of epochs it becomes possible to study their apparent acceleration both due to bending and due to intrinsic changes in Lorentz factor. It appears that real changes in Lorentz factors for jet features play an important role in observed accelerations 32 . Whether these accelerations reflect changes in the underlying flow is unclear; however a tendency for jet features with increasing speed to appear closer to the base of their jets than features with decreasing speed may be evidence of corresponding changes in the flow speed on these length scales 32 . Finally, the ejection of multiple components along differing position angles in jets, when studied over very many epochs, allows the production of stacked, time-averaged images of the jet morphology 24 , suggesting a possible new way of studying jet opening angles and collimation.

\footnotetext{
${ }^{\mathrm{b}}$ Although note that component 5 in figure 2 is non-ballistic, indicating that it has changed its trajectory since ejection to be about 8 degrees to the south, relative to a ballistic trajectory from the base of the jet.
} 

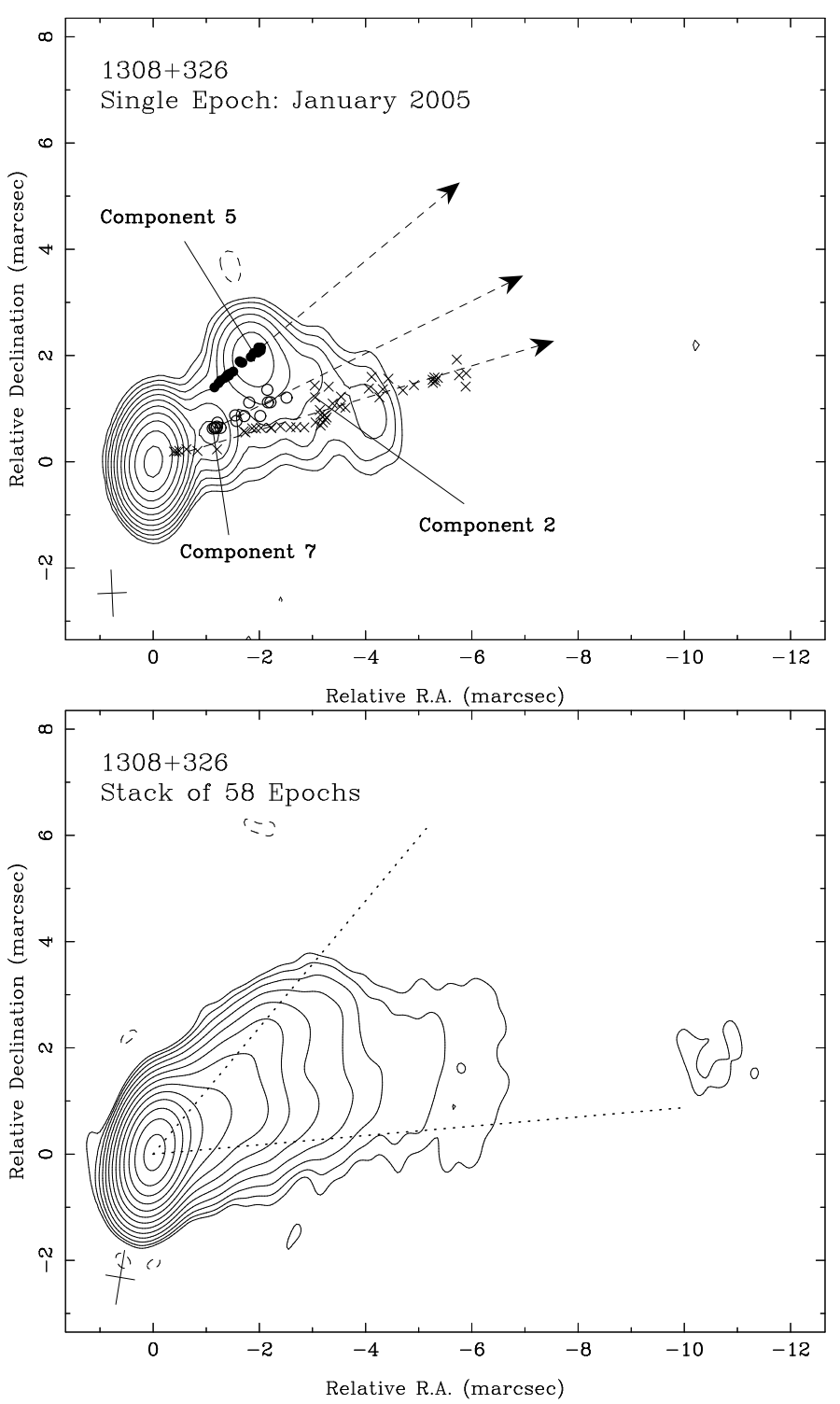

Fig. 2. $15 \mathrm{GHz}$ VLBA Images of the quasar $1308+326$ at $z=0.997$, both in a single MOJAVE epoch, top panel, and stacked over 58 MOJAVE and archival epochs processed by the MOJAVE program, bottom panel (http://www.physics.purdue.edu/astro/MOJAVE/). The apparent motion of three components are over-plotted on the top panel to illustrate the outward nature of the motions. Components 2 and 7 are consistent with ballistic trajectories while component 5 is nonballistic by about 8 degrees, indicating that it has changed its motion since being ejected from the core. The bottom panel has dotted lines indicating the approximate opening cone revealed in the stacked image. 


\section{Acknowledgments}

I would like to thank all the members of the MOJAVE program including Matt Lister, Yuri Kovalev, Ken Kellermann, Hugh Aller, Margo Aller, Tigran Arshakian, Andrei Lobanov, Tuomas Savolainen, Anton Zensus, Eduardo Ros, Matthias Kadler, Neil Gehrels, Julie McEnery, Marshall Cohen, Alexander Pushkarev, and Talvikki Hovatta. The MOJAVE program is funded at Purdue University through National Science Foundation grant AST-0807860 and NASA-Fermi grant NNX08AV67G. D. Homan was funded by National Science Foundation grant AST-0707693.

\section{References}

1. Meier, D. L., Koide, S., \& Uchida, Y., Science, 291, 84 (2001).

2. Vlahakis, N., Königl, A., The Astrophysical Journal, 605, 656 (2004).

3. Sikora, M., Begelman, M. C., Madejski, G. M., \& Lasota, J.-P., The Astrophysical Journal, 625, 72 (2005).

4. Marscher, A. P., Jorstad, S. G., D’Arcangelo, F. D., et al., Nature, 452, 966 (2008).

5. Vermeulen, R. C., \& Cohen, M. H., The Astrophysical Journal, 430, 467 (1994).

6. Lister, M. L. \& Marscher, A. P., The Astrophysical Journal, 476, 572 (1997).

7. Cohen, M. H., Lister, M. L., Homan, D. C., Kadler, M., Kellermann, K. I., Kovalev, Y. Y., \& Vermeulen, R. C., The Astrophysical Journal, 658, 232 (2007).

8. Kovalev, Y. Y., et al., The Astrophysical Journal, Letters, 696, L17 (2009).

9. Lister, M. L., Homan, D. C., Kadler, M., Kellermann, K. I., Kovalev, Y. Y., Ros, E., Savolainen, T., \& Zensus, J. A., The Astrophysical Journal, Letters, 696, L22 (2009).

10. Piner, B. G., Pant, N., \& Edwards, P. G., The Astrophysical Journal,723, 1150 (2010).

11. Marscher, A. P., Jorstad, S. G., Larionov, V. M., et al., The Astrophysical Journal, Letters, 710, L126 (2010).

12. Marscher, A. P., \& Gear, W. K., The Astrophysical Journal, 298, 114 (1985).

13. Hughes, P. A., Aller, H. D., \& Aller, M. F., The Astrophysical Journal, 341, 54 (1989).

14. Gómez, J. 2005, Real versus Simulated Relativistic Jets in Future Directions in High Resolution Astronomy eds. J. Romney and M. Reid, ASP Conference Proceedings Vol.340, 13

15. Hughes, P. A., Aller, M. F., \& Aller, H. D., The Astrophysical Journal, 735, 81 (2011).

16. Homan, D. C., Ojha, R., Wardle, J. F. C., Roberts, D. H., Aller, M. F., Aller, H. D., \& Hughes, P. A., The Astrophysical Journal, 549, 840 (2001).

17. Kellermann et al., The Astrophysical Journal, 609, 539 (2004).

18. Jorstad, S. G., et al., The Astronomical Journal, 130, 1418 (2005).

19. Piner, B. G., Mahmud, M., Fey, A. L., \& Gospodinova, K., The Astronomical Journal, 133, 2357 (2007).

20. Britzen, S., et al., Astronomy and Astrophysics, 484, 119 (2008).

21. Lister, M. L., Cohen, M. H., Homan, D. C., et al., The Astronomical Journal, 138, $1874(2009)$.

22. Lister, M. L., \& Homan, D. C., The Astronomical Journal, 130, 1389 (2005).

23. Homan, D. C., \& Lister, M. L., The Astronomical Journal, 131, 1262 (2006).

24. Lister, M. L., et al., The Astronomical Journal, 137, 3718 (2009).

25. Kellermann, K. I., Vermeulen, R. C., Zensus, J. A., \& Cohen, M. H., The Astronomical Journal, 115, 1295 (1998).

26. Zensus, A., Ros, E., Kellermann, K. I., Cohen, M. H., and Vermeulen, R. C., The Astronomical Journal, 124, 662 (2003).

27. Kovalev, Y. Y., et al., The Astronomical Journal, 130, 2473 (2005). 
28. Jorstad, S. G., Marscher, A. P., Mattox, J. R., Aller, M. F., Aller, H. D., Wehrle, A. E., \& Bloom, S. D., The Astrophysical Journal, 556, 738 (2001).

29. Fey, A. L., Clegg, A. W., \& Fomalont, E. B., The Astrophysical Journal, Supplement, 105, 299 (1996).

30. Ojha, R., Homan, D. C., Roberts, D. H., et al., The Astrophysical Journal, Supplement, 150, 187 (2004).

31. Lister, M. L. et al. in preparation.

32. Homan, D. C., Kadler, M., Kellermann, K. I., et al., The Astrophysical Journal, 706, 1253 (2009).

33. Abraham, Z., \& Carrara, E. A., The Astrophysical Journal, 496, 172 (1998).

34. Wehrle, A. E., Piner, B. G., Unwin, S. C., Zook, A. C., Xu, W., Marscher, A. P., Teräsranta, H., and Valtaoja, E., The Astrophysical Journal, Supplement, 133, 297 (2001)

35. Jorstad, S. G., Marscher, A. P., Lister, M. L., Stirling, A. M., Cawthorne, T. V., Gómez, J.-L., \& Gear, W. K., The Astronomical Journal, 127, 3115 (2004).

36. Caproni, A., \& Abraham, Z., The Astrophysical Journal, 602, 625 (2004).

37. Lobanov, A. P., \& Roland, J., Astronomy and Astrophysics, 431, 831 (2005).

38. Stirling, A. M., et al., Monthly Notices of the Royal Astronomical Society, 341, 405 (2003).

39. Pushkarev, A. B., Kovalev, Y. Y., Lister, M. L., \& Savolainen, T., Astronomy and Astrophysics, 507, L33 (2009).

40. Hovatta, T., Valtaoja, E., Tornikoski, M., Lähteenmäki, A., Astronomy and Astrophysics, 494, 527 (2009). 